\title{
Traduire
}

Revue française de la traduction

\section{Les mécanismes de la confiance en traduction - aspects relationnels}

\section{Nicolas Froeliger}

\section{(2) OpenEdition}

Journals

Édition électronique

URL : http://journals.openedition.org/traduire/975

DOI : $10.4000 /$ traduire. 975

ISSN : 2272-9992

\section{Éditeur}

Société française des traducteurs

\section{Édition imprimée}

Date de publication : 1 mars 2008

Pagination : 24-39

ISSN : 0395-773X

\section{Référence électronique}

Nicolas Froeliger, «Les mécanismes de la confiance en traduction - aspects relationnels », Traduire

[En ligne], 216 | 2008, mis en ligne le 01 mars 2008, consulté le 19 avril 2019. URL : http://

journals.openedition.org/traduire/975; DOI : 10.4000/traduire.975 


\title{
Les mécanismes de la confiance en traduction - aspects relationnels*
}

\author{
Nicolas Froeliger \\ Université Paris Diderot (Paris 7) \\ Master professionnel ILTS \\ (Industrie de la langue et traduction spécialisée)
}

Telle école de traduction réputée proposait naguère, en formation continue, un cours de gestion du stress. Telle autre enseigne à ses étudiants comment se comporter face à un éventuel employeur (tant qu'à faire, exhiber plutôt une bouteille de Vittel qu'une canette de coca). Telle entreprise hésite fortement à embaucher la candidate la plus douée au motif que cette dernière fait figurer dans son curriculum vitae la pratique du djembé et des claquettes. Telle autre arrêtera son choix, à l'issue du premier entretien, sur la candidate qui porte un tout petit sac à main, garantie d'un sens aigu de l'organisation. Une troisième n'engagera que des diplômés de telle école, bien que ses membres en aient des souvenirs très mitigés et entretiennent avec elle des relations qui frisent l'exécrable. Très souvent, des clients demandent à leurs fournisseurs réguliers les coordonnées de traducteurs qu'ils s'abstiendront ensuite de contacter. Tel jeune diplômé brillant, accueilli dans un collectif de traducteurs par un ancien enseignant, s'y révèle incapable de formuler une phrase cohérente. Telle relation professionnelle s'interrompt un jour sans que, jamais, demandeur ou fournisseur ait eu l'occasion de se plaindre. Telle étudiante, enfin, rendra sa première préparation en s'excusant pratiquement d'exister : "j'espère que ça n'est pas trop catastrophique ", avant d'expliquer,

* Le présent article a initialement été publié en 2005, dans le numéro 2 de la revue en ligne "The Journal of Specialised Translation " : http://www.jostrans.org/issue02/art_froeliger.php 
pressée de questions, que, depuis un certain stage, "la perspective de rendre un travail [la] glace d'horreur. "

Tout traducteur professionnel est habitué à ces manifestations plus ou moins bizarres par lesquelles la confiance - ou son contraire - montre l'une de ses facettes. C'est la matière de multiples anecdotes, qui marquent l'appartenance culturelle à un corps de métier avant tout disparate. Bien conscients que la science n'est pas faite d'une accumulation de vignettes, nous pensons qu'il y a là plus que de l'anecdotique : pour nous, la confiance est un fil conducteur qui organise et conditionne la pratique de la traduction. Sans elle, pas de marché, pas de traducteurs qui durent, pas de textes efficaces. Il s'agit donc d'en dresser la cartographie : qu'entend-on par confiance, en général ; quels agencements naissent, en traduction, de la confiance en soi ou de son absence ; comment s'établit, ou pas, la confiance dans une relation traducteur-demandeur et comment faire naître ce mode de relation compte tenu de la discrétion qui est le lot naturel de la profession?

\section{Le kit de la confiance}

La confiance est à la mode. Ces dernières années, elle a fourni au monde de l'édition deux gros tirages (Fukuyama, 1995, et Peyrefitte, 1995) et une multitude de publications plus ciblées (voir Thuderoz, Mangematin et Harrison, 1999, pour une bibliographie détaillée). Nous retiendrons de cette notion, qu'il faut bien qualifier de molle, la définition de Mayer et al. (1995, p. 712) : il s'agit de «la volonté délibérée d'être vulnérable aux actions d'une autre partie fondée sur l'espérance que celle-ci accomplira une action importante pour la partie qui accorde sa confiance, indépendamment de la capacité de cette dernière à surveiller ou contrôler l'autre partie ". Reprenons ces divers éléments. Avec quoi fabrique-t-on de la confiance dans le contexte professionnel ?

- Avec du pluriel : il faut être au moins deux (ou en mesure de se dédoubler) pour se faire confiance. Dans tout acte de confiance, il y 
a un mouvement ou une interrogation regardant l'autre, et donc un engagement, une mise en jeu de sa propre identité.

- Avec de l'inconnu : si l'on place sa confiance (ou pas) en quelqu'un, c'est toujours quant à un objet tiers, à réaliser ou à s'abstenir de réaliser : saura-t-il en répondre ?

- Avec de la réciprocité : la confiance est un contrat. Si une des deux parties seulement remplit ses obligations, il y aura inéluctablement rupture.

- Avec du temps : cette concrétisation du contrat de confiance se déploie dans une dimension temporelle, dans un suspens. La cavalerie finira toujours par arriver avant la fin de la dernière bobine, moyennant quoi nous nous sentirons autorisés à recommander ce film à nos proches : "vous verrez, vous ne serez pas déçus".

- Avec du silence : si je dis "faites-moi confiance" ou une variante de cette formule (" je serai parfaitement franc avec vous", " je suis quelqu'un de foncièrement honnête ", " avec moi, il n'aura aucun problème ", etc.), j'éveillerai immanquablement la méfiance de mon interlocuteur.

Ces dimensions, on les retrouve dans la traduction : là aussi, il faut franchir une barrière, s'aventurer dans un domaine partiellement inconnu pour l'exécutant ou pour le destinataire (une langue, une culture, un savoir, une information) en faisant taire sa subjectivité, en s'engageant à rendre en temps voulu un texte conforme à un cahier des charges (implicite ou explicite), moyennant une rémunération. On rencontre aussi ces composantes dans le fonctionnement de l'économie monétaire (ne parle-t-on pas de monnaie fiduciaire ?) ou financière (une introduction en Bourse est un appel à la confiance des investisseurs). Dans tous les cas, il y a pari. Il y a aussi garantie : celle de l'État pour les emprunts des collectivités publiques ou parapubliques, la munificence des sièges sociaux pour les banques, les jeans taille basse et les casquettes portées à l'envers pour les rappeurs, les costumes sombres et les chaussures bicolores pour les mafiosi... Les 
ethos professionnels sont différents, mais l'effet recherché est le même : certifier qu'il n'y a pas tromperie sur la marchandise, ou sur l'effet à attendre. Sans doute parce qu'il joue sur le rapport entre représentation et profondeur, ce thème a fait l'objet de nombreux films, dont le modèle le plus achevé est sans doute L'Arnaque (The Sting) de George Roy Hill (1973). Dans cette œuvre mémorable, on remarquera que l'arme la plus efficace des deux protagonistes, bien plus que leur sens achevé du décorum, est tout simplement leur sourire.

\section{La confiance en soi}

C'est justement la confiance en soi rayonnant sur ces visages qui nous semble faire le plus défaut à beaucoup de traducteurs : n'est pas Paul Newman ou Robert Redford qui veut. Il est en effet rare qu'un traducteur se sente l'égal de ses interlocuteurs : si quelqu'un a tort, c'est obligatoirement lui, si quelque chose cloche dans le texte rendu, c'est encore de sa faute. L'autre a forcément raison. On le constate en particulier dans le contact avec les experts : très souvent, il faut faire appel à un spécialiste du domaine, impliqué ou non dans la relation commerciale, pour débrouiller les fils les plus complexes d'un texte. Malheureusement, cette instance de référence peut très bien se tromper : par ignorance du texte, par confusion du contexte, parce que sa méconnaissance de la langue l'incite à une attitude naïve ou parce que la question posée n'est pas celle qu'il faudrait (en particulier lorsqu'elle porte sur un point de vocabulaire considéré hors du raisonnement qui le porte et le justifie). Notre traducteur s'en rend généralement compte, mais aura souvent tendance à suivre l'avis dont, pourtant, il doute : manque de confiance en soi.

On observe le même comportement face aux erreurs factuelles figurant dans le texte original : on les identifie, on s'interroge et on les retranscrit avec une fidélité teintée de mauvaise conscience... Tout le monde sait qu'un traducteur de textes pragmatiques peut difficilement survivre sans se tenir au courant de l'actualité et sans entretenir un minimum de bagage culturel. Pourtant, face à une contradiction entre 
ce qu'il sait et ce qu'il doit traduire (" [...] the number of sovereign states increased from 55 in 1900 to 192 in 2000 while the number of democracies increased from zero to 120 respectively. " : cherchez l'erreur...), son jugement sera comme suspendu et il pourrait se laisser traduire n'importe quelle idiotie : sacralisation de la fonction d'auteur. Les auteurs des textes de départ sont, eux aussi, faillibles, ce qui devrait finalement être plutôt rassurant pour tout le monde. Avoir confiance en soi, c'est aussi ne pas accorder une confiance absolue aux autres !

Le manque de confiance en soi est responsable de deux problèmes stylistiques classiques : le mot à mot et la sous-traduction. Pourquoi a-t-on tendance à produire, en traduction, un calque du texte original ? Par démission devant le réel : c'est trop compliqué pour moi, alors je mets les mots sans chercher à comprendre, et Dieu reconnaîtra les siens. Attitude théologique(1) $:$ il y a quelqu'un, quelque part, qui comprend ce que ces mots signifient. Un être parfait - en tout cas bien plus parfait que ne saurait l'être un simple traducteur... Si l'on se laisse penser de la sorte, il devient logique de traduire la trace, dans le plus grand respect de l'incompréhensible ordonnancement originel, en espérant que quelqu'un d'autre, un jour, parviendra à résoudre le mystère. C'est une démission du traducteur devant sa propre aptitude à comprendre et à restituer un raisonnement. Il faut bien sûr partir du principe opposé : ce qu'un être humain a pu écrire, un autre doit forcément pouvoir le traduire dès lors qu'il dispose des outils nécessaires et d'une part de confiance en soi. Un exemple : dans un texte sur l'avenir des réseaux de communication tout optique(2), on apprend qu'il sera bientôt possible de regrouper des données à transmettre sous forme de salves optiques (optical bursts), avant de préciser "In the future, optical burst

(1) On peut y voir une survivance de la racine latine du mot confiance : fides, qui a donné la foi.

(2) C'est-à-dire où tous les composants électroniques ont laissé la place à des matériels optiques. Aujourd'hui, une grande partie des réseaux à fibres optiques sont encore mixtes, et relèvent de ce que l'on appelle l'optoélectronique. 
data will be switched in order to obtain higher efficiency of the network operation. The burst data has granularity between stream and packet data."

La première phrase ne présente pas de difficulté particulière(3). Arrêtons-nous sur la seconde. Stream correspond ici au flux de données, c'est-à-dire à une forme de transmission en continu, par opposition à celle par paquets (packet), familière aux réseaux Internet classiques. Quant à granularity (granularité, ou parfois modularité), c'est un concept qui renvoie au degré de fragmentation d'un système ou d'un programme, et donc, occasionnellement, à sa possibilité, en intelligence artificielle, de changer des éléments sans avoir à modifier l'ensemble. À ce stade, le problème ne concerne plus le vocabulaire : nous avons la trace, il reste à créer le lien. C'est ici qu'intervient la véritable compétence du traducteur, non pas dans la terminologie, mais dans la mise en système : "Selon ce principe, les salves de données pourront être transmises aussi bien en flux que par paquets (granularité). " Le terme granularité se retrouve ainsi entre parenthèses et en fin de phrase, alors qu'il était en vedette dans le texte original, mais c'est parce qu'il est redondant par rapport au reste $\mathrm{l}$ la granularité, c'est ici ce qui existe entre (between) la transmission en flux et par paquets. Ici, il est impératif de s'éloigner de l'original pour rendre l'intention de la phrase. Traduire, en ce sens, c'est faire un pari sur la justesse de ce que l'on croit comprendre. Dans un tel cas, on peut toujours réduire l'aléa par la connaissance de la langue et du domaine, ainsi que par les contacts avec les spécialistes. Mais cet aléa ne sera jamais nul. Nousmêmes, qui avons dû traduire ce passage, ne pouvons pas affirmer avec certitude que notre solution est parfaite. Ce que nous savons, c'est qu'elle a été déduite d'un contexte et d'une documentation. Et aussi, parce que le traducteur est un être prudent, qu'elle a été validée par un spécialiste. Mais cela ne fait pas une certitude absolue : il existe toujours un résidu, une part dévolue à la confiance que le traducteur place

(3) «À l'avenir, on pourra procéder [à la transmission] par commutation de données sous forme de salves optiques, ce qui permettra une exploitation plus efficiente du réseau. " 
en lui-même - et qui conditionne la confiance que ses clients placent en lui. Il ne faut jamais rendre un texte en s'excusant, quoi que l'on puisse penser de ce que l'on a écrit.

C'est le même défaut de confiance qui conduit certains traducteurs à systématiquement sous-traduire (rendre l'anglais huge par considérable, conséquent ou important, par exemple), à n'employer, quel que soit le style du texte de départ, que des clichés, poncifs, stéréotypes et expressions toutes faites : surtout pas de vagues! Autre manière de traiter une traduction avec des pincettes, on peut généraliser les formules servant à mettre les éléments de sens à distance les uns des autres (en matière de, pour ce qui est, le fait de...). Le traducteur doit être invisible, c'est vrai, mais certains prennent ce conseil un peu trop au pied de la lettre, s'imaginant qu'il leur faut purger le texte de toute vitalité - et au passage d'une partie de son sens comme de son pouvoir communiquant. Qu'on le veuille ou non, c'est une profession qui attire les timides, ces lointains continuateurs d'une figure drolatique imaginée par Dezsö Kosztolànyi : le " traducteur cleptomane ", qui prélevait sa dîme sur les textes originaux :

Comprenez-vous ce qu'avait fait notre malheureux confrère [...] ? Il avait tout simplement volé les bijoux de famille de la comtesse Éléonore comme il avait dépouillé avec une légèreté tout aussi impardonnable le compte Vitsislav, pourtant si sympathique, de ses mille cinq cents livres, ne lui en laissant que cent cinquante, et soustrait deux des quatre lustres de cristal de la salle de bal, et subtilisé vingt-quatre des trente-six fenêtres de l'antique château rescapé de tant d'orages. J'étais pris de vertige(4).

C'est évidemment tout le contraire qui est nécessaire : si le traducteur doit s'effacer, c'est pour mieux faire ressortir son texte. Dans l'idéal, le demandeur ne doit pas pouvoir reconnaître l'auteur de la traduction(5). Pour que l'intention initiale soit correctement rendue, la figure du traducteur doit se faire imperceptible, « comme à la limite de la mer

(4) Dezsö Kosztolànyi, Le Traducteur cleptomane et autres histoires, traduit du hongrois par Ádám Peter et Maurice Regnault, Viviane Hamy, 1994, p. 15.

(5) C'est ce qui permet de traduire un texte à plusieurs. 
un visage de sable(6). " Ce que nous produisons, c'est du neutre, mais l'on se méprend souvent sur cette obligation de neutralité. Elle signifie l'abandon (temporaire) de sa propre subjectivité : un traducteur n'a pas d'amour-propre ; il n'a que le désir de rendre des textes acceptables. Il faudrait donc remplacer la figure du traducteur cleptomane par celle du traducteur artiste-décorateur : le grand problème, ici, c'est d'être dans le ton, afin que la traduction ne se voie pas, afin que le texte produise seul son effet. Il faut pour cela se faire suffisamment confiance pour abdiquer, devant son ordinateur, une part de soi-même. Il y a là un travail à mener qui n'a que peu de points communs avec le talent ou la maîtrise de la langue et des techniques, mais qui n'en est pas moins indispensable pour faire des traducteurs qui durent.

\section{Les paradoxes de la clôture}

Le jeu de la confiance se joue également entre un demandeur et un traducteur. Pourquoi un donneur d'ordre va-t-il arrêter son choix sur tel prestataire plutôt que sur tel autre?

- Rarement à cause du prix proposé : dans certains cas, un tarif que l'on pense compétitif, et donc attractif, suscitera au contraire la méfiance de son interlocuteur. La somme pour laquelle on monnaie ses services trahit aussi la confiance que l'on a en sa propre valeur. Et comment se fier à quelqu'un qui n'a apparemment pas confiance en lui-même?

- Rarement à cause de la publicité ou du démarchage : dans une profession de timides, les approches ouvertement commerciales apparaissent vite suspectes.

Ces deux premières stratégies se révèlent néanmoins efficaces lorsqu'elles sont pratiquées par des agences de traduction dont le souci est avant tout de faire du chiffre et lorsqu'elles s'adressent non pas à des praticiens, mais à des gestionnaires, les acheteurs d'un grand groupe,

(6) Michel Foucault, Les Mots et les choses, une archéologie des sciences humaines, Gallimard, Paris, 1966, p. 398. 
par exemple, qui raisonnent en termes de budget global : la fonction traduction me coûte tant ; sur ce total, je dois pouvoir économiser tel pourcentage en accordant l'exclusivité des traductions à telle agence. Sur le terrain, il n'est pas rare que les donneurs d'ordre contournent ce type d'accords lorsqu'ils ont besoin de textes sur lesquels ils puissent vraiment compter : il suffit alors de faire disparaître le mot " traduction " de la facture.

Hormis ces deux cas de figure, il faut s'en remettre au bouche-àoreille : c'est par quelqu'un qui a déjà confiance que se propage la confiance. La circulation des noms peut ainsi emprunter les circuits les plus incongrus... Quoi qu'il en soit, ce métier que l'on dit solitaire fonctionne en fait essentiellement en réseau et les relations professionnelles s'y établissent par cooptation. D'où un esprit d'école assez prononcé - et parfois savamment entretenu par les formations ellesmêmes.

Sans aller jusqu'à affirmer que le secteur de la traduction est organisé selon le schéma des sociétés initiatiques, on peut observer qu'il a ses mécanismes de cooptation, ses épreuves (selon le principe que l'on apprend par la souffrance), ses rites de passage (celui du client impossible que l'on adresse aux jeunes confrères avec une sollicitude à peine tempérée par un zeste de curiosité sadique), ses invocations (la norme ISO 9002), ses signes de reconnaissance (la relation des confrères aux manifestations du réel, cruciales lorsqu'on a affaire à des textes pragmatiques), son éthique confraternelle (la réciprocité, aussi appelée renvoi d'ascenseur, qui fait qu'il est très rare qu'un traducteur cherche à accaparer le client d'un autre - pas seulement parce que ces professionnels sont de bonnes âmes, mais plus prosaïquement parce qu'ils se couperaient ainsi avec certitude du reste de leur communauté...), ses blagues pour initiés (le général Staff, par exemple, identifié par Pierre Vidal-Naquet en 1974 et popularisé depuis par le bouche-à-oreille), ses tabous (rendre un texte en retard, voire, très souvent, se permettre de demander un délai supplémentaire, ou encore claironner que l'on est parfaitement bi-, tri- ou quadrilingue et que l'on saura traduire 
dans tous les sens) et ses coups de poignard ("C'est un très bon traducteur, peut-être un peu littéraire...»).

Attardons-nous sur deux paradoxes de la confiance liés aux manifestations de cet ethos professionnel. Le premier a trait à un comportement implicite : encore aujourd'hui, la traduction reste dans une large mesure une profession régie par des contrats oraux (dans la tradition du fameux Texas cattle deal handshake). En général, ces contrats sont respectés - et bien des demandeurs seraient outrés qu'on leur réclame un bon de commande : « comment cela, vous ne me faites pas confiance !? ". Parfois non : en traduction aussi, il y a des faillites. On y croise même d'occasionnels escrocs. En l'absence de commande écrite, il est alors parfaitement vain de chercher à faire valoir ses droits(7)... Comme la plupart des traducteurs l'ont appris à leurs dépens, il faut donc accorder sa confiance avec discernement. De même, il est très rare que l'on demande à un traducteur de signer un engagement de confidentialité : on présuppose qu'au contact d'informations sensibles, il saura rester neutre (qu'il s'abstiendra d'acheter des actions de telle entreprise qui s'apprête à faire une annonce spectaculaire, par exemple(8)). Les initiés sont nombreux ; les délits sont rares, du moins chez les traducteurs.

Examinons un autre paradoxe. Le téléphone sonne. Nous décrochons. Les premiers mots de notre interlocutrice sont les suivants : «Bonsoir, vous avez un jardin ? ”. Ce n'est pas la représentante d'un pépiniériste qui nous parle, mais une donneuse d'ordre, ancienne traductrice ellemême, qui publie un magazine sur... le jardinage, on s'en doutait.

(7) Que l'on se rassure, en cas de règlement judiciaire, un bon de commande n'est pas non plus d'une grande aide, puisque les prestataires de services extérieurs sont parmi les derniers créanciers à obtenir satisfaction, après l'État, les organismes de recouvrement sociaux, les salariés et les fournisseurs de biens matériels. La pratique, de plus en plus répandue, à ne travailler que sur bon de commande nous semble donc - hélas - simplement destinée, une fois encore, à se rassurer.

(8) À maintes reprises, nous avons traduit, en externe des textes - financiers, en particulier - très sensibles sans jamais que l'on songe à nous demander une telle garantie, alors même que tous les salariés des banques et sociétés de bourse qui nous confiaient ces textes étaient astreints à signer de tels documents. 
Dans son esprit, il est inconcevable de produire des textes qui fonctionnent dans ce domaine si l'on vit en appartement. Ridicule, évidemment. Notamment parce que ce n'est pas précisément le genre d'entrée en matière qui inspire confiance : cela aussi, on apprend à le repérer. Mais révélateur, aussi : pour beaucoup, on ne peut traduire correctement que si l'on fait partie du même monde que ses mandants. Il faut avoir été reçu et accepté. D'où des mécanismes tendant à susciter des microsociétés à l'intérieur desquelles tomberont les barrières de la défiance : la mafia, les Auvergnats de Paris, les tenants du Pernod contre ceux du Ricard (il est des nôtres...), les traducteurs... Or, il ne faudrait tout de même pas oublier que la fonction première de cette profession consiste à faire franchir les frontières de toutes natures. Ce que l'on nous demande, implicitement, c'est d'être à la fois dedans et dehors : montrer que l'on fait partie d'un même monde et briser les limites de ce monde. Pour être lui-même, le traducteur doit ainsi endosser à la fois la tunique de Romulus et celle de Remus : celui qui trace les limites et celui qui les enjambe. La mythologie nous apprend que cela ne finit pas toujours très bien. Mais le bon sens - et la théorie du nationalisme - nous enseignent que cette dialectique est constitutive des fonctions d'intermédiation :

Même les sociologues les plus éminents [...] affirment que ces minorités ont une double norme, celle qu'elles observent dans leur groupe et une autre, amorale et instrumentaliste, qui vaut pour le monde extérieur. Elles ont en effet une double norme, mais qui fonctionne exactement à l'inverse. Leur attitude à l'égard du monde traditionnel consistait à rendre des services précis ou à fournir une marchandise précise. Leur réputation et leur revenu dépendaient entièrement du fait quils accomplissaient ces tâches avec fiabilité et ils étaient connus pour leur fiabilité professionnelle( $(9)$.

Qu'est-ce qui était en jeu, à l'époque prémoderne où certaines fonctions (l'armée, la haute administration, la circulation de l'argent) étaient réservées à des catégories sociales précises, qui avaient pour

(9) Ernest Gellner, Nations et nationalisme, traduit de l'anglais par Bénédicte Pineau, Bibliothèque historique Payot, Paris, 1989, 208 pages, p. 149. 
obligation de s'y cantonner ? Tout simplement la rationalité des contrats : l'assurance d'obtenir ni plus ni moins que ce que l'on demandait. Dans son mode de fonctionnement, le secteur de la traduction pragmatique est héritier de cette configuration. Et il serait sans doute très éclairant d'étudier l'histoire de cette profession sous cette grille de lecture, comme cela a été fait, par exemple, pour la traduction des textes littéraires ou religieux : la neutralité du traducteur préexistait-elle à la rationalité qui caractérise l'époque moderne et son résidu contemporain, la traduction des textes pragmatiques?

\section{Une dialectique de la discrétion}

Nous avons vu, plus haut, que la confiance, en général, ne se gagnait pas directement : on ne dit pas "faites-moi confiance", mais on installe ses locaux (ou on se fait domicilier) dans un quartier cher et réputé. Ce mécanisme fonctionne habituellement par affichage : il s'agit d'exhiber les qualités qui vont susciter la confiance. Les traducteurs se heurtent ici à une difficulté : la discrétion qui leur incombe aux yeux du reste du monde. En effet, un bon traducteur est un traducteur invisible, puisque le texte d'arrivée est considéré comme de qualité lorsqu'il ne vient à personne l'idée qu'il s'agit d'une traduction. Toute la stratégie de communication initiale d'un hebdomadaire composé à $90 \%$ de traductions a procédé selon cet escamotage : "Courrier international : l'essentiel de la presse mondiale en français ». L'élision tout à fait réfléchie(10) du participe passé " traduite " faisait peu de cas de l'amour-propre des traducteurs, mais elle a montré son efficacité.

De fait, beaucoup de ces derniers se plaignent de n'être pas reconnus, de n'intervenir qu'en bout de chaîne, c'est-à-dire, très souvent, au moment où il ne reste plus suffisamment de temps pour produire un texte potable. Dans le Code des impôts, cette profession figurait jadis, dit-on, au dernier article, celui des " autres professions ", où elle

(10) Nous en avons obtenu la confirmation auprès d'un des fondateurs de cet hebdomadaire. 
côtoyait les montreurs d'ours. Dans la nomenclature des conventions collectives, elle était rattachée à la sérigraphie (un texte spécifique est censé y mettre prochainement bon ordre). Les professionnels s'en plaignaient et s'en plaignent. À juste titre. Pourtant, on peut se demander s'il n'y a pas là le signe, paradoxal, d'une confiance : le traducteur, aux yeux des demandeurs non avertis, c'est une boîte noire : quelqu'un qui, malgré tous ces handicaps, saura produire dans les délais impartis et sans se faire remarquer un texte qui fonctionne. D'où les cours de gestion du stress - et un fort sentiment de victimation. Une touche de masochisme, parfois aussi(11).

Ainsi, la confiance est là, mais ses manifestations sont mal vécues. Pour tenter de sortir de cette pénible situation, il faut tenter de se hisser au rang d'interlocuteur à part entière. Comment faire ? Une fois un contact professionnel établi, il est raisonnable de l'entretenir. Certains traducteurs pensent qu'il est pour cela indispensable de ne jamais dire non : subordination totale au donneur d'ouvrage. D'autres estiment qu'il faut savoir doser le refus. Nous ne trancherons pas entre les tenants de la fidélité absolue et ceux de la jalousie tactique. L'une et l'autre armes ont toutefois leurs limites. Mieux vaut donc, dans un cas comme dans l'autre, chercher à mieux se comprendre. Il importe pour cela d'apprendre à expliciter les non-dits d'une demande : vous me réclamez quarante pages en trois jours dans un domaine que je découvre. Je peux à la rigueur vous les fournir dans les délais, mais vous comprenez bien qu'il m'est impossible de vous garantir une qualité irréprochable... Il existe deux grandes variables d'ajustement dans la négociation d'une traduction : le temps (qui cesse d'être une variable dès que la mission est acceptée) et la qualité(12). Cette pratique du

(11) La confiance aussi fonctionne selon le principe de la boîte noire, et non comme on l'entend parfois selon celui de la transparence : avoir confiance, c'est estimer qu'il n'est pas nécessaire d'aller regarder de plus près. D'où l'importance donnée, dans les sociétés de confiance (Peyrefitte, 1995), c'est-à-dire, peu ou prou, les économies libérales, aux commissaires aux comptes et autres cabinets de vérification et d'audit.

(12) Le prix (tarif d'urgence, en l'occurrence) n'est en général qu'une pauvre variable de consolation... 
" oui, mais ", qui a connu son heure de gloire en politique, a pour principal avantage de placer le demandeur face à ses responsabilités : elle rééquilibre la relation, et seules les relations équilibrées sont durables.

Et cette confiance-là, typiquement, sera rompue à l'arrivée d'un interlocuteur nouveau ou lorsque le traducteur commettra l'erreur de montrer qu'il en sait plus que son demandeur : il a tout à gagner à rechercher l'égalité, mais tout à perdre à affirmer sa supériorité, y compris face à ceux dont le métier n'est ni de comprendre ni de savoir. Même chose vis-à-vis des spécialistes : le traducteur a besoin d'eux pour déchiffrer les arcanes les plus subtils de ses textes, mais il doit d'abord les convaincre qu'il est capable d'y comprendre quoi que ce soit. Le plus simple consiste alors à partir d'une erreur et d'un illogisme du texte de départ pour poser une question faussement naïve : « je ne suis pas bien certain de comprendre l'articulation entre ces deux points... ». La relation quitte ainsi le domaine technique pur - où le traducteur ne peut qu'être en situation d'infériorité. La confiance est une rencontre ; elle prospère entre égaux. En s'attachant à éduquer ses clients ou ses demandeurs, le traducteur peut ainsi accéder (à leurs yeux) à une part d'existence qui lui permettra de travailler dans de meilleures conditions et d'accéder à un semblant de reconnaissance.

Pour fonctionner à bon escient, la confiance doit être équilibrée par le doute : il n'est jamais nuisible de douter, à dose raisonnable, de soimême et des autres. Car nous sommes dans un jeu perpétuellement mouvant : les niveaux de compétence sont changeants et un excellent traducteur peut très vite baisser sa garde et se mettre à produire des textes catastrophiques. C'est un lieu commun, et nous ne le rappelons que pour mémoire. Nous pensons que sous la confiance, réflexive ou réciproque, se décide quelque chose de bien plus décisif : la confiance (ou son absence), c'est ce qui va déterminer le lieu d'exercice de 
l'autorité. La question de confiance, en traduction, c'est finalement qui décide ?

- Si c'est l'auteur, représenté faute de mieux par le texte de départ, le traducteur aura raison de laisser intactes ses erreurs et inexactitudes.

- Si c'est le demandeur, (même lorsque celui-ci n'a aucune compétence pour juger de la validité d'un choix), le traducteur n'aura qu’à courber la tête devant le traitement fait à son texte en aval de la traduction (phénomène fréquent lorsqu'on travaille pour la presse).

- Si c'est un expert mal questionné ou un dictionnaire, le traducteur sera tranquillisé : certes, il aura écrit (parfois en connaissance de cause) un certain nombre d'inexactitudes, mais au moins, il sera couvert. L'emblème de cette profession devrait être le parapluie.

- Si c'est le traducteur envers et contre tous, le produit rendu pourrait n'avoir qu'un lointain rapport avec l'intention du texte initial.

Chacune de ces hypothèses mène donc à une dégradation de la fonction de communication. Ce qui importe, au contraire, c'est de parvenir à situer l'opération et les indispensables postures à adopter par chacun des intervenants dans un terrain neutre, accessible à tous et sur lequel aucun ne puisse affirmer un droit exclusif : un lieu commun, précisément. Ce terrain, c'est la langue et la logique en tant que patrimoines universels. Faire accepter collectivement que le sens n'appartient à personne en propre, mais se construit, se perfectionne et se partage, c'est avoir une chance, en premier lieu, de se comprendre et, en second lieu, de produire des textes qui fonctionnent. Tous les intervenants doivent être détenteurs d'une part d'autorité, mais d'une part seulement, et c'est au point de convergence de ces quatre influences que s'établira la traduction la moins mauvaise possible. Mettre en place une telle configuration est la tâche première du traducteur. Dans cette démarche essentiellement laïque, il possède quelques facilités puisque son travail consiste à comprendre et à expliciter ce qui est obscur. C'est un atout dont il aurait tort de ne pas faire usage.

Nous sommes conscients, en ayant décrit ces différentes postures, de n'avoir encore rien dit, ou presque, sur l'essentiel, c'est-à-dire sur les 
textes. Qu'est-ce qu'un texte digne de confiance ? À quels signes le reconnaîtra-t-on ? Qu'est-ce que faire foi, pour une traduction ? Ces questions feront l'objet d'une prochaine contribution, mais il fallait commencer par le commencement, c'est-à-dire par les facteurs psychologiques et par les savoir-faire tacites qui structurent la traduction en tant que discipline.

nf@eila.univ-paris-diderot.fr

Diplômé de l'ESIT, traducteur technique au sein de la société Architexte et auteur d'une thèse sur Thomas Pynchon, Nicolas Froeliger enseigne l'anglais et la traduction de textes pragmatiques à l'Université Paris VII. Ses travaux antérieurs ont porté sur le cliché, l'interdisciplinarité, la forme en traduction technique, la question des personnages en traduction de textes pragmatiques et les postures nécessaires à l'exercice de la traduction. Il s'intéresse globalement aux passerelles susceptibles d'exister entre savoir technique, culture générale et culture littéraire.

\section{Références}

Fukuyama, Francis (1995). Trust: the Social Virtues and the Creation of Prosperity, New York: Free Press.

Ladmiral, Jean-René (1994). Traduire : théorèmes pour la traduction. Paris, Gallimard, Tel.

Mayer, R.C., Davis, J. H. et Schoorman, F. D., 1995, "An integrative model of organizational trust", Academy of Management Review, Vol. 20, pp. 709-734.

Peyrefitte, Alain (1995). La Société de confiance. Paris, Odile Jacob.

Thuderoz, Christian, Mangematin, Vincent et Harrison, Denis (1999). La Confiance. Paris, Gaëtan Morin.

Vidal-Naquet, Pierre (1974). «Le général Staff ». Le Monde, p. 16. Cet article est repris dans Ladmiral (1994). 